\section{$\underset{\substack{\text { hommes } \\ \text { \& migrations }}}{ }$}

\section{Hommes \& migrations}

Revue française de référence sur les dynamiques

migratoires

$1324 \mid 2019$

Religion et discrimination

\title{
Autorités publiques, laïcité et discriminations religieuses
}

\section{Christine Pauti}

\section{(2) OpenEdition \\ Journals}

Édition électronique

URL : https://journals.openedition.org/hommesmigrations/8337

DOI : 10.4000/hommesmigrations.8337

ISSN : 2262-3353

Éditeur

Musée national de l'histoire de l'immigration

Édition imprimée

Date de publication : 1 janvier 2019

Pagination : 57-64

ISBN : 978-2-919040-44-5

ISSN : $1142-852 X$

Référence électronique

Christine Pauti, « Autorités publiques, laïcité et discriminations religieuses », Hommes \& migrations [En ligne], 1324 | 2019, mis en ligne le 01 janvier 2022, consulté le 06 janvier 2022. URL : http:// journals.openedition.org/hommesmigrations/8337 ; DOI : https://doi.org/10.4000/ hommesmigrations.8337 


\section{Autorités publiques,}

laïcité

et discriminations

religieuses

\section{Christine Pauti,}

maître de conférences en droit public, université Paris 1 Panthéon-Sorbonne.

Si la politique de l'État en matière de laïcité est censée assurer la non-discrimination à raison de la religion, son application actuelle tend parfois à contredire cet objectif. Au point d'aboutir à un paradoxe: la France, l'un des seuls pays laïques en Europe, est celui dans lequel la discrimination religieuse est ressentie le plus intensément. La gestion de ce paradoxe échoit de plus en plus aux collectivités territoriales. Le niveau local est, en effet, souvent celui où se joue la mise en œuvre des principes de laïcité et de non-discrimination. Or les interprétations différentes de ces principes selon les territoires sont aujourd'hui porteuses de risques ou, au contraire, d'espoirs quant à leur garantie pour la population Française ou étrangère qui y réside.

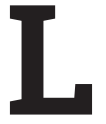

es deux premiers articles de la loi du 9 décembre 1905 sur la séparation des Églises et de l'État reflètent le principe de laïcité, qui porte sur le respect de la liberté de conscience et du libre exercice des cultes de chacun, d'une part, et le principe de neutralité de l'État, d'autre part. Au regard du service public, le principe de laïcité comporte une obligation de neutralité religieuse pesant non seulement sur les fonctionnaires et les agents publics, mais également sur les bâtiments publics. La non-discrimination apparaît comme un élément constitutif du principe de neutralité. En écho à la «théorie des apparences» chère à la Cour européenne des droits de l'homme, nul ne doit ressentir un quelconque sentiment de discrimination et exclusion de sa commune, de son département ou de sa région, et plus largement de l'État, en étant identifié ou rattaché à une tradition ou à une croyance religieuse.

Le principe de non-discrimination à raison de la religion constitue l'un des piliers du principe d'égalité rappelé dès la Révolution française. Et c'est au lendemain de la Seconde Guerre mondiale, où la discrimination religieuse par des régimes ayant "tenté d'asservir et de dégrader la personne humaine» avait atteint les summums de l'atrocité, 
qu'est proclamé solennellement dans le préambule de la Constitution du 27 octobre 1946 que «tout être humain, sans distinction de race, de religion ni de croyance, possède des droits inaliénables et sacrés», parmi lesquels la liberté religieuse. Le principe de non-discrimination a depuis lors investi le champ mondial - avec notamment la Déclaration sur l'élimination de toutes formes d'intolérance et de discrimination fondées sur la religion ou la conviction proclamée par l'Assemblée générale des Nations unies le 25 novembre 1981 - et, en particulier, européen avec l'article 14 de la Convention européenne des droits de l'homme ${ }^{1}$ et le droit de l'Union européenne depuis l'adoption du Traité d'Amsterdam en 1997.

En 2012, dans son rapport sur la discrimination religieuse, la Commission européenne classait la France comme dernier élève en matière de perception qu'avaient ses habitants de la discrimination religieuse: 66 \% d'entre eux considéraient que la discrimination sur des fondements religieux était généralisée dans le pays². On se retrouve ainsi face à ce paradoxe: la France, quasiment le seul pays européen laïque, est celui dans lequel la discrimination religieuse est ressentie le plus intensément...

Au moment même où la politique de lutte contre les discriminations se renforce, puisqu'un nouveau critère de discrimination lié à la précarité sociale a été inséré à l'article 225-1 du Code pénal en juillet 2016 et que la loi du 18 novembre 2016 de modernisation de la justice du XXIe siècle crée une action de groupe dans le domaine de la lutte contre les discriminations, la France persiste à ne pas ratifier le Protocole $n^{\circ} 12$ à la Convention européenne des droits de l'homme qui consacre l'interdiction générale de la discrimination.

Par ailleurs, force est de constater aujourd'hui une montée de l'intolérance religieuse, soulignée notamment par la Commission nationale consultative des droits de l'homme dans son rapport de 2015 sur La lutte contre le racisme, l'antisémitisme et la xénophobie et par la Commission européenne contre le racisme et l'intolérance (Ecri), dans son rapport sur la France du 8 décembre 2015.

Si une part importante des discriminations religieuses sévit dans le secteur privé, les autorités publiques, qu'elles soient nationales ou locales, sont également au cœur de nombreuses problématiques religieuses porteuses de potentielles discriminations. Soumises au principe de laïcité et donc de neutralité, sous le regard médiatique, ces autorités sont particulièrement attentives au respect du principe de non-discrimination. Elles sont d'ailleurs plus sévèrement punies quand elles commettent une discrimination. C'est également le cas quand cette dernière est commise dans un lieu accueillant du public ${ }^{3}$.

Cependant, alors même que le principe de laïcité est censé préserver la non-discrimination, la politique de l'État engendre aujourd'hui des conséquences inattendues, voire défavorables à ce principe. En outre, les collectivités territoriales, par la marge de manœuvre dont elles disposent en ce domaine, sont en passe de devenir des acteurs privilégiés de la garantie du droit à la non-discrimination religieuse.

\section{Les conséquences paradoxales de la politique de l'État en matière de laïcité}

L'évolution de l'interprétation du concept de laïcité ainsi que la réglementation en ce domaine ont des effets paradoxaux sur le principe de non-discrimination et son application dans le service public et, plus largement, dans l'espace public. Si le principe de laïcité est affecté d'une telle ambiguïté aujourd'hui, c'est sans doute en grande partie en raison de l'incertitude

\footnotetext{
1. Art. $14 \mathrm{CEDH}$ : «La jouissance des droits et libertés reconnus dans la présente Convention doit être assurée, sans distinction aucune, fondée notamment sur le sexe, la race, la couleur, la langue, la religion, les opinions politiques ou toutes autres opinions, l'origine nationale ou sociale, l'appartenance à une minorité nationale, la fortune, la naissance ou toute autre situation. »

2. Special Eurobarometer 393, Discrimination in the EU in 2012, European Commission, novembre 2012, p. 49.

3. Art. 225-1 Code pén. : " Constitue une discrimination toute distinction opérée entre les personnes physiques lou les personnes morales en vertu de l'alinéa suivant] sur le fondement [...] de leur appartenance ou de leur non-appartenance, vraie ou supposée, à [...] une religion déterminée. » L'article 225-2 du Code pénal prévoit des peines pouvant atteindre trois ans d'emprisonnement et 45000 euros d'amende. Lorsqu'un refus discriminatoire de fournir un bien ou un service est commis dans un lieu accueillant du public ou aux fins d'en interdire l'accès, les peines peuvent aller jusqu'à cinq ans d'emprisonnement et 75000 euros d'amende.

Art. 432-7 Code pén. : «La discrimination définie aux articles 225-7 et 225-7-7, commise à l'égard d'une personne physique ou morale par une personne dépositaire de l'autorité publique ou chargée d'une mission de service public, dans l'exercice ou à l'occasion de l'exercice de ses fonctions ou de sa mission, est punie de cinq ans d'emprisonnement et de 75000 euros d'amende lorsqu'elle consiste $7^{\circ}$ À refuser le bénéfice d'un droit accordé par la loi ; $2^{\circ}$ À entraver l'exercice normal d'une activité économique quelconque. »
} 
qui plane sur la signification exacte du principe de neutralité et de ses contours, laquelle entraîne inévitablement des conséquences sur l'autre facette du principe de laïcité - la liberté religieuse de chacun -, ouvrant la voie à davantage de risques de discriminations.

\section{Si une part importante des discriminations} religieuses sévit dans le secteur privé, les autorités publiques, qu'elles soient nationales ou locales, sont également au cœur de nombreuses problématiques religieuses porteuses de potentielles discriminations.

Le principe de laïcité est aujourd'hui invoqué par une multiplicité d'acteurs avec des significations floues et souvent contraires. La compréhension juridique traditionnelle du principe est notamment remise en cause par l'avènement d'un courant prônant une «nouvelle laïcité» à laquelle est censé se soumettre un nombre sans cesse croissant de personnes au sein tant de structures publiques que privées. Sont concernés les stagiaires en formation continue dans les groupements d'établissements publics locaux d'enseignement (Greta ${ }^{4}$ ) proposant des formations continues pour adultes, les accompagnateurs de sorties scolaires ${ }^{5}$, les étudiants à l'université ${ }^{6}$, les salariés de structures en charge de la petite enfance ${ }^{7}$, les salariés d'organismes privés associés au service public ${ }^{8}$, les usagers de centres d'hébergement et de réinsertion sociale...

Dans son rapport de 2015 sur La lutte contre le racisme, l'antisémitisme et la xénophobie, et dans la continuité de son avis sur la laïcité du 26 septembre 2013, la Commission nationale consultative des droits de l'homme met en garde "contre les dévoiements incessants d'une "nouvelle laïcité" qui tend à exiger et à imposer une neutralisation religieuse». Elle porte notamment un regard critique sur la Charte de la laïcité qui tend "à réduire au mutisme la liberté religieuse et la diversité des croyances ${ }^{9}$ ».

Dans son rapport de 2017, le Défenseur des droits constate quant à lui un «contexte particulièrement instable marqué par un brouillage de la notion de laïcité [...] émergence d'une nouvelle conception de la laïcité conduisant à étendre progressivement l'obligation de neutralité proscrivant les signes religieux, en particulier musulmans, à l'entreprise privée et à l'accès aux biens et services privés ${ }^{10} 》$.

On tente ainsi de déployer le principe de laïcité non seulement à d'autres secteurs que le secteur public, mais également, au sein même du service

4. Voir TA Paris, 27 avril 2009, n0905233/9 : est illégale l'annulation de l'inscription d'une stagiaire voilée par un président d'un Greta car il s'est fondé exclusivement sur les dispositions de la loi du 15 mars 2004, "sans justifier de l'existence, en l'espèce, de troubles à l'ordre public ou d'un comportement de la requérante conférant au port de son foulard le caractère d'un acte de pression ou de prosélytisme »; TA Paris, 2 octobre 2010, n0905232; CAA Paris, 12 octobre 2015, $n^{\circ} 14 \mathrm{PA} 00582$ : «Dans les conditions dans lesquelles se déroulait cet enseignement, les stagiaires du Greta étaient amenés à rencontrer les élèves de ce lycée; que la présence simultanée, dans l'enceinte d'un même établissement, de ces élèves, qui sont soumis, en application des dispositions de l'article L. 147-5-7 du code de l'éducation, à l'interdiction de port de signe manifestant ostensiblement une appartenance religieuse, et d'une stagiaire du Greta portant un tel signe, était dès lors, dans les circonstances de l'espèce, de nature à troubler l'ordre dans cet établissement. » 5. Voir la circulaire Chatel $n^{\circ} 2012-056$ du 27 mars 2012 (NOR: MENE1209011C) ; TA Montreuil, 22 novembre 2011, n 1012015 , Osman (JCP A, 2011, 2384 ; AJDA 2012.163, note S. HennetteVauchez; D. 2012. 72, obs. M.-C. de Montecler, note A.-L. Girard ; AJCT 2012. 105, obs. P. Rouquet; D. adm. 2/2012, pp. 42-46, note A. Taillefait) ; CE avis, 23 décembre 2013 (non publié) ; TA Nice, 9 juin 2015, $n^{\circ} 1305386$ (AJDA 2015.1933, note C. Brice-Delajoux : RJPF 9/2015, pp. 22-24, note J. Couard).

6. Voir délibération Halde $n^{\circ} 2008-194,29$ septembre 2008 : le comportement d'un enseignant qui interdit l'accès à ses cours à des élèves voilées "caractérise manifestement une différence de traitement en raison du port du foulard islamique et ne peut en aucun cas être justifié par le principe de la laïcité. En effet, la loi n²004-228 du 15 mars 2004 [...] ne s'applique pas à l'université. Par ailleurs, conformément à l'article 871-7 du code de l'éducation, les étudiants disposent de la liberté d'expression qu'ils exercent à titre individuel ou collectif, en veillant à ne porter atteinte ni aux activités d'enseignement et de recherche, ni à l'ordre public ».

7. Voir Cass., Ass. plen. n¹3-28.369, 25 juin 2014 (D. 2014. 1386; AJDA 23/2014.1293 et 32/2014.1842, note S. Mouton et T. Lamarche ; RFDA 2014. 954, note P. Delvolvé ; RTDC 2014. 620, obs. J. Hauser) : rejet du pourvoi formé par la directrice adjointe de la crèche Baby Loup contre l'arrêt de la Cour d'appel de Paris, licenciée par son employeur à la suite du refus d'ôter son voile.

8. Cass., $n^{\circ} 12-11690,19$ mars 2013, Mme X. c/CPAM de Seine Saint Denis (JCP A, 29 avril 2013, pp. 51-56, note J.-B. Vila; LPA 127/2013, pp. 7-14, note H. Peschaud) : les principes de neutralité et de laïcité sont applicables à l'ensemble des services publics, y compris lorsque ceux-ci sont assurés par des organismes de droit privé. Voir néanmoins CJUE, 14 mars 2017, C-157/1 et C-188/15 ; Cass. Soc., 22 novembre 2017, n²484/1319.855 : l'interdiction du port de signes religieux n'est licite que dans la mesure où le règlement intérieur de l'entreprise ou une note de service d'effet équivalent prévoit une «clause de neutralité » qui doit être générale et indifférenciée et ne peut être appliquée qu'aux salariés en contact avec la clientèle.

9. $\mathrm{CNCDH}$, La lutte contre le racisme, l'antisémitisme et la xénophobie, Paris, La documentation française, 2015, p. 196.

10. Défenseur des droits, Rapport annuel d'activité, Paris, Défenseur des droits, 2017, p. 95. 
public, on essaie d'imposer la neutralité à certains usagers, en principe non concernés par cette facette du principe de laïcité.

Cette extension des frontières de la laïcité s'est concrètement traduite par l'adoption de la loi du 15 mars 2004 encadrant le port de signes ou de tenues manifestant une appartenance religieuse dans les écoles, collèges et lycées publics. Ainsi, alors que, d'ordinaire, les spécificités d'un service public - en l'espèce celles liées à la vulnérabilité des élèves en raison de leur âge - servent à réaffirmer le principe de neutralité imposé aux agents, elles ont ici permis de justifier une entorse à la conception traditionnelle du principe de laïcité, restreignant la liberté religieuse de ses usagers et entraînant des conséquences négatives sur le principe de non-discrimination.

\section{Les répercussions sur les discriminations religieuses dans le service public}

En raison d'une mauvaise connaissance - ou d'une compréhension délibérément biaisée - tant du principe de laïcité que du champ d'application de la loi du 15 mars 2004, des usagers du service public subissent parfois des discriminations liées à leur religion de la part d'autorités publiques: ainsi, dans une délibération n²006-132 du 5 juin 2006, la Haute Autorité de lutte contre les discriminations et pour l'égalité (Halde) condamne la discrimination dont a été victime un Sikh exclu d'une salle d'audience car il portait le turban, alors qu'il n'avait eu «aucune attitude indigne et irrespectueuse, et n'a[vait] porté aucun trouble à la sérénité de la justice». Une récente affaire de la Cour européenne des droits de l'homme, Lachiri c. Belgique du 18 septembre 2018, concerne l'exclusion d'une salle d'audience d'une requérante - partie civile dans une affaire de crime passionnel dans laquelle son frère avait été tué - car elle refusait d'enlever son hijab ${ }^{11}$. La Cour constate que la façon dont $\mathrm{M}^{\mathrm{me}}$ Lachiri s'est comportée lors de son entrée en salle d'audience n'était pas irrespectueuse ou ne constituait pas - ou ne risquait pas de constituer une menace pour le bon déroulement de l'audience. La Cour juge donc que la nécessité de la restriction litigieuse ne se trouve pas établie et que l'atteinte portée au droit de $\mathrm{M}^{\mathrm{me}}$ Lachiri - simple citoyenne, ne représentant pas l'État - à la liberté de manifester sa religion n'était pas justifiée dans une société démocratique. Cette même Cour, s'agissant de l'exclusion d'une avocate voilée en Espagne, ayant déclaré la requête irrecevable, n'a malheureusement pas pu se prononcer sur le fond ${ }^{12}$. De même, en 2006, la Halde a considéré que le fait de refuser à une femme l'accès à la cérémonie de remise du décret relatif à sa naturalisation organisée dans l'enceinte de la préfecture en raison du port du voile revêtait un caractère discriminatoire ${ }^{13}$. Par ailleurs, aucun texte n'étend le principe de neutralité aux élus qui, pour la plupart, notamment les conseillers municipaux, ne sont pas des agents publics et disposent d'une pleine liberté d'expression religieuse.

Lors des élections régionales de 2010, le juge des référés a estimé qu'en enregistrant une liste comprenant une femme portant le voile islamique, l'administration n'avait pas porté une atteinte grave et manifestement illégale à la liberté de conscience des requérants car «il y avait lieu de combiner ces principes, d'une part, avec la liberté individuelle de la candidate, d'autre part, avec le droit de celle-ci de se présenter à une élection ${ }^{14} »$. Par la suite, le Conseil d'État a refusé de renvoyer la question prioritaire de constitutionnalité relative aux dispositions du Code électoral mises en cause, en jugeant «que la circonstance qu'un candidat à une élection affiche son appartenance à une religion est sans incidence sur la liberté de choix des électeurs et ne met pas en cause l'indépendance des élus; qu'aucune norme constitutionnelle, et notamment pas le principe de laïcité, n'impose que soient exclues du droit de se porter candidates à des élections des personnes qui entendraient, à l'occasion de cette candidature, faire état de leurs convictions religieuses ${ }^{15} \gg$.

Une fois élus, les membres d'assemblées délibérantes jouissent de la liberté de conscience et de la liberté d'expression. Ainsi, en 2010, la Cour de

11. CourEDH, Hagar Lachiri c. Belgique, 18 septembre 2018, n³413/09 (Gaz. Pal. 32/2018, note C. Berlaud; Dalloz actualités, 26 septembre 2018, note V.-O. Dervieux).

12. CourEDH, Barik Edidi c. Espagne, 19 mai 2016, n²1780/13.

13. En revanche, ce refus ne peut être considéré comme le refus d'un droit accordé par la loi au sens de l'article 432-7 du Code pénal, son décret lui ayant été remis en mains propres (Halde, Délibération 5 juin 2006, n²006-131). Mais le Conseil d'État a validé le rejet de la naturalisation d'une Algérienne qui avait refusé, pour des raisons religieuses, de serrer la main à un représentant préfectoral et à un élu lors de sa cérémonie d'accueil dans la nationalité française, « dans un lieu et à un moment symboliques », ce qui « révélait un défaut d'assimilation », en application des dispositions de l'article 21-4 du Code civil (CE, 11 avril 2018, $n^{\circ} 412462$ ).

14. CE, $1^{\text {er }}$ mars $2010, n^{\circ} 337079$ (RJPF, 6/2010, pp. 13-14, note E. Putman).

15. CE, 23 décembre 2010, n³37899, Assoc. Arab Women's Solidarity Association France et a. 
cassation a jugé coupable de discrimination un maire qui avait privé une élue de l'exercice de son droit de parole lors d'une réunion du conseil municipal en raison du port par cette dernière d'une croix, alors même qu'il n'était «nullement établi, qu'en l'espèce, le port d'une croix [...] ait été un facteur de trouble». La Cour a estimé "qu'aucune disposition législative [...] ne permet au maire d'une commune, dans le cadre des réunions du conseil municipal, lieu de débats et de confrontations d'idées, d'interdire aux élus de manifester publiquement, notamment par le port d'un insigne, leur appartenance religieuse ${ }^{16} »$. Outre les glissements du sens de la laïcité, la réglementation peut également avoir des effets sur le principe de non-discrimination.

\section{Les conséquences de la réglementation sur les religions et la laîcité}

À côté des inégalités prévues dans les textes, certaines réglementations sur la laïcité peuvent, en pratique, entraîner des effets discriminatoires à l'encontre de croyants de confessions déterminées. D'un point de vue géographique tout d'abord, la loi du 9 décembre 1905 portant séparation des Églises et de l'État ne s'applique pas uniformément sur le territoire de la République. Cette adaptation territoriale de la réglementation cultuelle, conséquence de facteurs historiques et culturels, entraîne une inégalité entre les croyants en fonction de leur lieu d'habitation: en Alsace-Moselle, par exemple, où la loi de 1905 ne s'applique pas, un élève pourra, s'il le souhaite, recevoir des cours de religion à l'école ou choisir, dans certaines cantines, des repas halal.

Du point de vue des religions ensuite, il existe une inégalité de traitement juridique, due à l'existence de règles datant de l'époque où le culte catholique était ultra-majoritaire en France. Le statut de la propriété des édifices cultuels est emblématique. En effet, l'État et les collectivités financent l'entretien et la rénovation des lieux de cultes antérieurs à 1905: il s'agit donc principalement d'édifices chrétiens. Ceux construits après cette date, comprenant notamment davantage de mosquées et de synagogues, ne bénéficient pas de ces financements. Et l'inégalité persiste malgré une tentative de rééquilibrage opérée par le juge grâce à une lecture plus souple du principe de neutralité des collectivités territoriales ${ }^{17}$. Un traitement inégal n'est cependant pas forcément discriminatoire. En effet, en vertu du droit français, un traitement juridique peut être différent si les distinctions en rapport avec l'objet de la loi sont fondées soit sur des motifs d'intérêt général, soit sur des différences de situations objectives ${ }^{18}$. On peut néanmoins s'interroger en l'espèce pour savoir si les différences de situations sont telles qu'elles justifient, encore aujourd'hui, le traitement privilégié réservé aux catholiques. Au-delà d'une éventuelle discrimination en droit, certaines règles juridiques existantes sont potentiellement porteuses d'inégalité entre les croyants dans les faits.

La France a souvent été critiquée en raison de réglementations perçues comme discriminatoires à l'encontre des croyants de certaines confessions. Ont notamment pu être condamnées les interdictions du port de signes religieux sur les photographies d'identité et de la dissimulation du visage dans l'espace public. Sans compter la réglementation adoptée en 2004 sur les signes religieux à l'école qui, au-delà des élèves, emporte des risques de discrimination et de stigmatisation des femmes voilées et de la confession musulmane en général. Dans son rapport de 2010, l'Ecri recommandait aux autorités françaises de procéder à une évaluation de la loi du 15 mars 2004 sur les signes religieux à l'école sous l'angle de la discrimination indirecte et de la stigmatisation ${ }^{19}$. Et, en 2015, le Comité des droits de l'homme jugeait que les effets de cette loi et de celle du 11 octobre 2010 sur l'interdiction de la dissimulation du visage dans l'espace public étaient contraires aux buts recherchés par le législateur car elles favoriseraient «le

16. Cass., crim., $1^{\text {er }}$ septembre $2010, n^{\circ} 10-80584$ (AJDP Dalloz, 11/2010. 506, note G. Royer ; Droit pénal, n 12, 2010, pp. 27-28, note M. Véron; JCP G Semaine Juridique, $n^{\circ}$ 49, 2010, pp. 2278-2280, note E. Dreyer).

17. Voir notamment les cinq décisions CE, 19 juillet 2011, Commune de Trélazé, n 308544 ; Fédération de la libre pensée et de l'action sociale du Rhône et M. P. n 308817 ; Communauté urbaine du Mans - Le Mans métropole, n 309161 ; Commune de Montpellier, $n^{\circ} 313518 ; M^{\text {me }} V . n^{\circ} 320796$ sur la conciliation entre intérêts publics locaux et loi du 9 décembre 1905 et notamment $M^{\text {me }} V$. $n^{\circ} 320796$ sur un bail emphytéotique administratif conclu entre une collectivité territoriale et une association cultuelle en vue de l'édification d'une mosquée (RFDA, 5/2011, pp. 967-986, note E. Geffray ; Revue Lamy des collectivités territoriales, 73/2011, pp. 10-15; AJDA 2014.124, note S. Hubac).

18. CE, Sect., 9 mars 1951, Société des concerts du Conservatoire (Rec., p. 151) ; CE, 10 mai 1974, Denoyez et Chorques (Lebon p. 274 ; AJDA, 1974, p. 298, chron. M. Franc et M. Boyon ; RDP, 1975, p. 467, note M. Waline; Rev. adm., 1974, p. 440, note F. Moderne; D. 1975, jur. p. 393, note P. Tedeschi) ; CC n 79-107 DC, 12 juillet 1979, Ponts à péages (JO, 13 juillet 1979 ; Rec., p. 31).

19. Ecri, $4^{e}$ rapport, 29 avril 2010, publié le 15 juin 2010, pp. 24-25. 
sentiment d'exclusion et de marginalisation de certains groupes » composant la société française ${ }^{\mathbf{2 0}}$.

Face aux répercussions de la politique de l'État en matière de laïcité sur le principe de nondiscrimination, l'analyse de la politique locale s'impose tant elle semble aujourd'hui représenter un véritable enjeu en la matière.

\section{La politique locale: un enjeu stratégique en matière de laïcité}

Les problématiques de laïcité et de non-discrimination religieuse semblent aujourd'hui davantage se cristalliser au niveau local car les collectivités disposent d'une marge de manœuvre non négligeable, génératrice de dangers tout autant que d'espoirs selon l'utilisation qui en est faite.

Les collectivités territoriales sont au cœur de bien des problématiques religieuses, tant par le biais de transferts de compétences que par celui de la clause de compétence générale, qui demeure désormais pour la seule commune: traditionnelle question des sonneries de cloches des églises, gestion des lieux et du patrimoine cultuels, financement de projets d'intérêt public local ou mise à disposition de locaux et d'équipements communaux, gestion des cimetières... De récentes initiatives témoignent d'une prise de conscience nouvelle de la sensibilité et de l'actualité de ces questions au niveau local: institution, en 2011, d'une conférence départementale de la liberté religieuse et d'un correspondant «laïcité»; publication au cours des dernières années de guides consacrés à la laïcité au sein des collectivités locales par l'Observatoire de la laïcité, le Centre national de la fonction publique territoriale (CNFPT), l'Association des maires de France (AMF) ou la Mairie de Paris... Le lien entre collectivités territoriales et principe de non-discrimination à raison de la religion est même explicitement assuré depuis 2003 à l'article $1^{\text {er }}$ de la Constitution qui proclame à la fois le caractère laïque de la République française, le principe d'égalité devant la loi de tous les citoyens sans distinction de religion, le respect de toutes les croyances ainsi que l'organisation décentralisée de la République.

Par ailleurs, quand bien même les règles sont nationales, c'est souvent au niveau local qu'elles reçoivent application, entraînant des variations géographiques dans leur mise en œuvre effective. On pense ici, par exemple, aux interprétations différentes données par les inspections d'académies à la question des mères voilées accompagnatrices de sorties scolaires qui avaient conduit la Halde en 2007 à recommander au ministre de l'Éducation nationale «de prendre toute mesure nécessaire afin d'assurer, selon les mêmes modalités sur l'ensemble du territoire, le respect du principe de non-discrimination ${ }^{21}$ ».

Le CNFPT a quant à lui organisé une offre de formation et une Offre nationale harmonisée sur le principe de laïcité. Cet objectif d'harmonisation témoigne bien des difficultés auxquelles sont confrontées les collectivités locales et d'une certaine territorialisation en la matière.

\section{La marge de modulation du droit à la non-discrimination à raison de la religion}

Si toutes les collectivités territoriales sont astreintes au respect de garanties incompressibles attachées au principe de neutralité, elles bénéficient d'une marge de manœuvre non négligeable dans le cadre des services publics locaux facultatifs, ce qui n'est pas exempt de conséquences sur le principe de non-discrimination.

Les collectivités locales, tout comme l'État, sont liées par le principe de neutralité qui a des implications diverses tant au niveau de l'accès aux services qu'à celui du respect de la libre expression religieuse des habitants. S'agissant du lien entre principe de neutralité et accès aux services de la collectivité, tout d'abord, à partir du moment où une commune crée un service public, même facultatif, elle ne peut interdire son accès, sans commettre de discrimination, pour des motifs tenant à la religion d'un usager. De même, le Défenseur des droits a jugé discriminatoire le refus, durant trois années successives, de louer une salle municipale à une association souhaitant célébrer la commémoration de la mort du Christ alors qu'elle en avait fait la demande un an à l'avance et que d'autres salles municipales étaient manifestement inoccupées ${ }^{22}$. Le Conseil d'État a quant à lui estimé que le refus de louer «une salle municipale afin de permettre d'accueillir la célébration de la fête de l'Aïd-el-Kebir porte une atteinte grave et manifestement illégale aux libertés de réunion et de culte» et

20. Comité des droits de l'homme, Observations finales concernant le cinquième rapport périodique de la France, 21 juillet $2015, \S 22$.

21. Halde, Délibération, 14 mai $2007, n^{\circ} 2007-117$.

22. Défenseur des droits, décision $n^{\circ}$ MLD-2013-6, 28 février 2013. 
a ordonné au maire de mettre le local à disposition de l'association ${ }^{23}$. Par un arrêt du $1^{\text {er }}$ mars 2005, la Cour de cassation a également jugé coupable de discrimination religieuse un maire qui réservait en priorité les créneaux du samedi après-midi pour se marier civilement aux personnes de confession catholique qui célébraient leur mariage ensuite à l'église ${ }^{24}$.

Par ailleurs, il y a quelques années, le ministère de l'Intérieur avait dû, par circulaire, rappeler aux préfets les règles du droit de l'urbanisme s'appliquant à la construction de lieux de culte ${ }^{25}$ pour faire face aux refus de permis de construire opposés par certains élus locaux à des associations cultuelles, pouvant s'apparenter à des discriminations.

Des élus ont été jusqu'à envisager d'interdire l'accès à leur commune en raison de la religion des futurs habitants. En septembre 2015, quelques maires de droite, répondant à l'appel du gouvernement, se sont ainsi portés volontaires pour accueillir des réfugiés, mais à la condition qu'ils soient chrétiens, car, selon eux, ils ne mettaient pas en danger la sécurité d'autrui... Le Premier ministre a dû rappeler que l'accueil des réfugiés ne pouvait se faire sur la base de leur religion, ce qu'a également indiqué l'ONU en novembre 2015 à l'adresse de certains responsables politiques américains et européens.

S'agissant du lien entre principe de neutralité et libre expression religieuse ensuite, en vertu de l'article 10 de la Déclaration des droits de l'homme et du citoyen de 1789: "Nul ne doit être inquiété pour ses opinions, même religieuses, pourvu que leur manifestation ne trouble pas l'ordre public établi par la loi.» Dans l'espace public et dans les services publics - hormis au sein des écoles, des collèges et des lycées depuis la loi du 15 mars 2004 -, les citoyens et les usagers ont le droit d'exprimer librement leurs convictions religieuses, y compris par le port de tenues vestimentaires ou de signes religieux, exclusion faite de la dissimulation de leur visage depuis la loi du 11 octobre 2010. Or, en juillet 2014, dans la commune de Wissous, des femmes voilées se sont vues refuser l'accès à un espace de loisirs sur le fondement d'une disposition de son règlement intérieur qui interdisait l'accès, dans un premier temps, à toute personne "ayant une tenue inappropriée ou contraire aux règles de décence ou le port de tout signe religieux», puis, dans un second temps, "aux personnes portant de manière ostentatoire des signes religieux susceptibles d'occasionner un trouble à l'ordre public (niqab ou burqa, croix ou étoile de dimension manifestement excessive)». Dans les deux cas, coupant court à la tentative de déployer la logique de la loi du 15 mars 2004 au-delà du domaine scolaire, le juge administratif a estimé qu'en l'absence de nécessités tirées du bon fonctionnement du service ou de l'ordre public, les dispositions litigieuses étaient «constitutive[s] d'une discrimination fondée sur la seule appartenance à une religion et port[aient] ainsi une atteinte grave et manifestement illégale à la liberté d'expression religieuse $e^{26}$ ». Comme l'a confirmé le Conseil d'État dans une décision du 26 août 2016 annulant des arrêtés similaires dans la commune de Villeneuve-Loubet, un maire ne peut se fonder sur d'autres considérations que le bon accès au rivage, la sécurité de la baignade ainsi que l'hygiène

\section{(1. S'agissant du lien entre principe de neutralité et libre expression religieuse ensuite, en vertu de l'article 10 de la Déclaration des droits de l'homme et du citoyen de 1789 : « Nul ne doit être inquiété pour ses opinions, même religieuses, pourvu que leur manifestation ne trouble pas l'ordre public établi par la loi. »}

et la décence sur la plage pour prendre ce type d'arrêté. En l'absence de risques avérés d'atteinte à l'ordre public, «l'émotion et les inquiétudes résultant des attentats terroristes [...] ne sauraient suffire à justifier légalement la mesure d'interdiction contestée $e^{27} \gg$. Et c'est justement parce qu'il a jugé que les conditions liées à l'ordre public étaient réunies que le tribunal administratif de Bastia a, par la suite, validé l'arrêté municipal réglementant la baignade sur la plage de $\operatorname{Sisco}^{28}$.

23. CE, ord. 23 septembre 2015, Association des Musulmans de Mantes sud, $n^{\circ} 393639$ (JCP A, n 40, 5 octobre 2015, act. 794, pp. 11-12).

24. Cass., crim., $1^{\text {er }}$ mars $2005, n^{\circ} 04-83556$, Joël $X$.

25. Circulaire NORINTA0500022C du 14 février 2005 du ministre de l'Intérieur, de la sécurité intérieure et des libertés locales adressée aux préfets relative à la construction d'édifices du culte, règles d'urbanisme et de construction.

26. TA Versailles, 12 juillet $2014 n^{\circ} 1404939$ et $n^{\circ} 1404959$ et TA Versailles, 12 août $2014 n^{\circ} 1405744$.

27. CE, ord. 26 août 2016, Ligue des droits de l'homme et autres et Association de défense des droits de l'homme Collectif contre I'islamophobie en France, $n^{\circ} 402742$ et n 402777 (D. 2016.1704; D. adm. 8/2016, p. 9 ; RFDA 6/2016, pp. 1227-1234, note P. Bon ; AJTC 2016, p. 508, obs. G. Le Chatelier)

28. TA Bastia, ord. 6 septembre 2016, Association Lique des droits de l'homme, $n^{\circ} 1600975$; CAA Marseille, 3 juillet 2017, n 17MA01337; CE, 14 février 2018, Ligue des droits de l'homme, $n^{\circ} 413982$. 
Si les collectivités locales sont ainsi liées par ces implications du principe de neutralité, en revanche, elles retrouvent une marge de manœuvre importante quand il s'agit de répondre à des exigences rattachées aux convictions religieuses des usagers dans l'organisation des services publics locaux facultatifs.

\section{Les aléas territoriaux du droit}

\section{à la non-discrimination dans les services publics locaux facultatifs}

Un usager ne peut pas exiger qu'un service public s'adapte à ses convictions religieuses. Il serait discriminatoire, car contraire au principe d'égalité, d'interdire à des usagers l'accès à un équipement public, par exemple à une piscine municipale, pendant ses heures d'ouverture au public pour satisfaire aux exigences de réservation de créneaux horaires non mixtes pour des motifs religieux d'autres usagers.

Le cas des cantines est emblématique. En vertu du principe de neutralité, les communes n'ont pas d'obligation de mettre en place de menus prenant en compte des prescriptions religieuses. Il est cependant fréquent que les cantines servent du poisson le vendredi et proposent des plats de substitution à la viande de porc. Mais le refus d'une collectivité territoriale d'adapter les repas en fonction des convictions religieuses n'est pas considéré comme discriminatoire. En revanche, dans une délibération n²03 du 2 octobre 2006, la Halde a estimé qu'un prestataire de services ayant «mis en place un dispositif permettant aux enfants de confession musulmane de bénéficier d'un substitut de repas à la viande de porc [...] constituerait une discrimination le fait de ne pas accorder la même possibilité aux enfants de religion hindouiste».

Ainsi, la prise en compte des souhaits des familles dépend de la libre appréciation de chaque collectivité territoriale, entraînant une différence de traitement en fonction du lieu d'habitation des familles: certaines communes, se rapprochant ce faisant des techniques canadiennes ou américaines, pourront faire le choix d'«aménagements raisonnables » en décidant d'offrir des menus adaptés aux convictions religieuses afin de rééquilibrer au profit de certains croyants une situation créant une discrimination indirecte. D'autres, au contraire, pourront choisir de refuser toute adaptation: conséquences de deux conceptions du principe d'égalité - égalité en droit (ou «formelle») et égalité substantielle (ou «réelle») - aucun de ces choix communaux, aux résultats pourtant diamétralement opposés, n'est a priori contraire aux principes de laïcité et de nondiscrimination, à moins que, derrière le refus de tout aménagement, ne soit décelée une discrimination fondée sur la religion. Par exemple, une municipalité Front national avait décidé, en invoquant des motifs de coût financier, de servir systématiquement du porc dans les cantines scolaires en supprimant les plats de substitution ${ }^{29}$

\section{(2) Le droit à la non-discrimination est donc interprété et appliqué différemment selon les territoires et la variété de leurs contextes historiques, politiques et sociaux.}

Le droit à la non-discrimination est donc interprété et appliqué différemment selon les territoires et la variété de leurs contextes historiques, politiques et sociaux. Le décalage est ainsi frappant entre la diffusion du droit universel à la non-discrimination religieuse et la marge de manœuvre et la responsabilité croissantes dont sont investies les collectivités locales en ce domaine, en l'absence - en partie inévitable - d'un cadre général ferme. Cette latitude offerte aux collectivités est tout à la fois porteuse d'insécurité juridique mais également d'opportunité pour une lecture plus ouverte du principe de laïcité que celle retenue sur le plan national.

Au final, la laïcité française, en particulier par la neutralité qu'elle impose à ses agents, constitue sans aucun doute un rempart solide contre certaines discriminations. Néanmoins, du fait de l'interprétation variable qui peut être donnée à ce concept, on peut s'interroger sur sa capacité à toujours réaliser un respect effectif de l'égalité dans le domaine religieux, tant au niveau national que local. On en revient au même paradoxe: alors que le principe de laïcité est censé intégrer en son sein le principe de nondiscrimination, son interprétation actuelle peut être porteuse de nouvelles discriminations..

29. TA Marseille, 26 novembre 1996, Mme Zitouni, Ghribi et a. c. Commune de Marignane, n96-3523 (LPA, 18 février 1997, p. 3, note G. Pélisier). 